\title{
Exploring Uncharted Terrain in Nature's Structure Space Using Capillary NMR Spectroscopy: 13 Steroids from 50 Fireflies
}

\author{
Matthew Gronquist, ${ }^{\dagger}$ Jerrold Meinwald, ${ }^{\ddagger}$ Thomas Eisner, ${ }^{\S}$ and Frank C. Schroeder* ${ }^{\star}$ \\ ${ }^{\dagger}$ Department of Chemistry, SUNY, College at Cortland, Cortland, New York 13045, \\ ${ }^{\ddagger}$ Department of Chemistry and Chemical Biology, Baker Laboratory, Cornell University, \\ Ithaca, New York 14853, and ${ }^{\S}$ Department of Neurobiology \& Behavior, Cornell \\ University, Ithaca, NY 14853.
}

\section{PART ONE. EXPERIMENTAL PROCEDURES AND SPECTROSCOPIC DATA FROM THE ANALYSIS OF L. ATRA}

\section{NMR probes and spectrometer}

All analyses were carried out using a VARIAN INOVA $600 \mathrm{MHz}$ NMR spectrometer, which was equipped alternatively with a $5 \mathrm{~mm}$ inverse-detection $\mathrm{HCN}$ probe or a $5 \mu 1$ PROTASIS/MRM capillary NMR probe. NMR spectra were acquired at $25^{\circ} \mathrm{C}$, using the standard pulse sequences provided by VARIAN. For each sample (sucrose or Lucidota atra sample) dqf-COSY, NOESY, HMQC, and HMBC spectra were acquired. For all spectra, phase-cycling was used for coherence selection. Gradients were used only for homospoil pulses during the relaxation delay. Acquisition times were set to $0.55 \mathrm{~s}$ (dqfCOSY) or $0.25 \mathrm{~s}$ (NOESY, HMQC, HMBC). For NOESY spectra, a mixing time of 600 ms was used. HMQC spectra were acquired without decoupling.

\section{CapNMR sample loading and recovery}

Solvents or sample solutions were drawn up into Hamilton syringes and then injected directly into the CapNMR probe inlet capillary. Prior to loading, the probe was rinsed with acetone and $\mathrm{D}_{2} \mathrm{O}$ (for the sucrose experiments) or methanol- $\mathrm{d}_{4}$ (for the L. atra samples) and subsequently flushed with air. Samples were dissolved in $50 \mu 1$ of deuterated solvent, filtered through a $2 \mu \mathrm{m}$ filter, evaporated to dryness in a conical vial, and carefully re-dissolved in 5.5-6 $\mu$ l of solvent. Special care was taken to ensure that all materials adhering to the walls of the vials were included. The resulting solution was then injected into the empty (air-filled) inlet capillary, immediately followed by injection of another $9 \mu 1$ of deuterated solvent, which served to push the sample solution into the flow cell. Any formation of air bubbles when drawing up sample and solvents has to be avoided, as air bubbles introduced into the flow cell severely impact overall performance. For sample recovery, the outlet capillary was attached to a recovery vial and the probe rinsed by injection of $50 \mu 1$ of deuterated solvent. Generally, more than $90 \%$ of a sample could be recovered, as determined from signal-to-noise comparison after re-injection of samples. 


\section{Sensitivity comparison on a $17.2 \mu$ g sucrose sample.}

For direct comparison of the $5 \mathrm{~mm}$ inverse-detection HCN probe with the CapNMR probe, $5 \mu \mathrm{l}$ of a $10 \mathrm{mM}$ sucrose solution in $\mathrm{D}_{2} \mathrm{O}$, corresponding to $17.2 \mu \mathrm{g}$ sucrose, was injected into the CapNMR probe as described above, followed by acquisition of dqfCOSY, HMQC, and HMBC spectra. Subsequently, $5 \mu \mathrm{l}$ of the $10 \mathrm{mM}$ sucrose solution were diluted into a volume of $200 \mu$ inside a Shigemi tube, followed by acquisition of the same three spectra with the $5 \mathrm{~mm} \mathrm{HCN}$ probe, using the same acquisition times, numbers of scans and numbers of increments as for the spectra acquired with the CapNMR probe. Signal-to-noise was four to five-fold better for the CapNMR spectra than for the spectra acquired using the HCN probe. Sections of the ${ }^{1} \mathrm{H}, \mathrm{HMQC}$, and HMBC spectra are attached.

\section{Preparation of Lucidota atra samples.}

Fifty individuals of $L$. atra were cut into pieces and then vigorously stirred in a 50:50 (v/v) mixture of methanol and dichloromethane $(150 \mathrm{~mL})$ for $48 \mathrm{hrs}$. The resulting solution was decanted from the remaining body parts, which were then rinsed with a second aliquot of methanol/dichloromethane followed by pure methanol. The combined extracts were concentrated, taken up in $100 \mathrm{~mL}$ of a 90:10 mixture of methanol and water $(\mathrm{v} / \mathrm{v})$, and then washed with two $100 \mathrm{~mL}$ portions of hexane. The resulting methanol/water solution was concentrated to give $88 \mathrm{mg}$ of an orange residue which was taken up in $750 \mathrm{ul}$ of methanol for HPLC injection. Preparative HPLC was carried out using an Agilent Series 1100 liquid chromatograph (10 x $250 \mathrm{~mm}$ Supelco $5 \mu$ ODS preparative column eluted at a flow rate of $3.8 \mathrm{~mL} / \mathrm{min}$ ) equipped with a diode array detector set to monitor wavelengths of $230 \mathrm{~nm}$ and $300 \mathrm{~nm}$. A gradient elution was used which started with a solvent composition of $10 \%$ acetonitrile and $90 \%$ water for three minutes, and then progressed to $25 \%$ acetonitrile by 30 minutes, and finally ramped to a composition of $100 \%$ acetonitrile by 40 minutes.

\section{Spectral data for $L$. atra samples}

${ }^{1} \mathrm{H}$ and ${ }^{13} \mathrm{C}$ NMR spectra were acquired in $\mathrm{CD}_{3} \mathrm{OD}$. Selected samples were further analyzed in $d_{6}$-DMSO as necessary to resolve overlapping signals (data not shown). Mass spectra were obtained using a Micromass Quattro I tandem mass spectrometer operated in positive-ion electrospray mode with direct infusion of each sample dissolved in a 50:50 (v/v) solution of methanol and water containing $1 \%$ formic acid. 


\section{Compound 1}

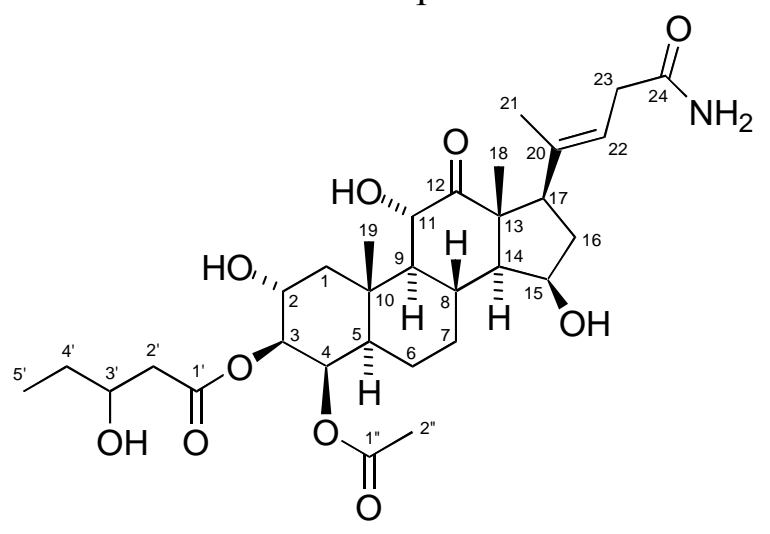

\begin{tabular}{|c|c|c|c|c|c|c|}
\hline $\begin{array}{c}\text { Carbon } \\
\text { No. }\end{array}$ & $\delta, \mathrm{ppm}$ & Proton No. & $\delta, \mathrm{ppm}$ & $J, \mathrm{~Hz}$ & $\begin{array}{c}\text { NOESY } \\
\text { Correlations }\end{array}$ & $\begin{array}{c}\text { HMBC } \\
\text { Correlations }\end{array}$ \\
\hline \multirow[t]{2}{*}{$\mathrm{C}-1$} & 47.6 & $1-\mathrm{H} \alpha$ & 1.45 & $\mathrm{~J}_{1 \alpha, 1 \mathrm{\beta}}=13.8, \mathrm{~J}_{1 \alpha, 2}=11$ & & \\
\hline & & $1-\mathrm{H} \beta$ & 2.60 & $\mathrm{~J}_{1 \beta, 2}=4.3$ & & \\
\hline $\mathrm{C}-2$ & 66.5 & $2-\mathrm{H}$ & 4.04 & $\mathrm{~J}_{2,3}=10.3$ & 19 & \\
\hline $\mathrm{C}-3$ & 78.2 & $3-\mathrm{H}$ & 4.69 & $\mathrm{~J}_{3,4}=3.8$ & & 1 , \\
\hline C-4 & 74.8 & $4-\mathrm{H}$ & 5.33 & $\mathrm{~J}_{4,5}=2$ & & $1 "$ \\
\hline C-5 & 48.7 & $5-\mathrm{H}$ & 1.57 & $\mathrm{~J}_{5,6 \mathrm{\alpha}}=2-3, \mathrm{~J}_{5,6 \mathrm{\beta}}=12.5$ & & \\
\hline \multirow[t]{2}{*}{ C-6 } & 25.7 & 6-H $\alpha$ & 1.41 & $\mathrm{~J}_{6 \alpha, 6 \beta}=12.5, \mathrm{~J}_{6 \alpha, 7 \alpha}=3-4$ & & \\
\hline & & $6-\mathrm{H} \beta$ & 1.61 & $\mathrm{~J}_{6 \beta, 7 \alpha}=12.5, \mathrm{~J}_{6 \beta, 7 \beta}=3-4$ & & \\
\hline \multirow[t]{2}{*}{$\mathrm{C}-7$} & 31.5 & $7-\mathrm{H \alpha}$ & 1.09 & $\mathrm{~J}_{7 \alpha, 7 \beta}=12.5, \mathrm{~J}_{7 \alpha, 8}=11.5$ & & \\
\hline & & $7-\mathrm{H} \beta$ & 2.17 & $\mathrm{~J}_{7,8}=3.5$ & & \\
\hline C-8 & 30.6 & $8-\mathrm{H}$ & 2.24 & $\mathrm{~J}_{8,9}=11.5, \mathrm{~J}_{8,14}=11.5$ & 18 & \\
\hline C-9 & 65.1 & $9-\mathrm{H}$ & 1.13 & $\mathrm{~J}_{9,11}=10$ & & \\
\hline $\mathrm{C}-10$ & 40.4 & & & & & \\
\hline $\mathrm{C}-11$ & 73.6 & $11-\mathrm{H}$ & 4.52 & & 19,18 & 12 \\
\hline $\mathrm{C}-12$ & 215.0 & & & & & \\
\hline$C-13$ & 57.6 & & & & & \\
\hline C-14 & 61.5 & $14-\mathrm{H}$ & 1.24 & $\mathrm{~J}_{14,15}=5.3$ & & \\
\hline C- 15 & 70.6 & $15-\mathrm{H}$ & 4.24 & $\mathrm{~J}_{15,16 \alpha}=6-7$ & & \\
\hline \multirow[t]{2}{*}{$\mathrm{C}-16$} & 39.3 & $16-\mathrm{H} \alpha$ & 1.97 & $\mathrm{~J}_{16 \alpha, 16 \beta}=14.5, \mathrm{~J}_{16 \alpha, 17}=8-9$ & 18 & \\
\hline & & $16-\mathrm{H} \beta$ & 2.30 & $\mathrm{~J}_{6 \beta, 17}=10-11$ & & \\
\hline $\mathrm{C}-17$ & 50.5 & $17-\mathrm{H}$ & 2.98 & & & $12,13,16$ \\
\hline $\mathrm{C}-18$ & 15.4 & $18-\mathrm{CH}_{3}$ & 1.16 & & 21 & $12,13,14,17$ \\
\hline C-19 & 16.0 & $19-\mathrm{CH}_{3}$ & 1.34 & & & $1,5,9,10$ \\
\hline $\mathrm{C}-20$ & 139.0 & & & & & \\
\hline $\mathrm{C}-21$ & 17.9 & $21-\mathrm{CH}_{3}$ & 1.75 & & 23 & $17,20,22$ \\
\hline $\mathrm{C}-22$ & 120.7 & $22-\mathrm{H}$ & 5.59 & $\mathrm{~J}_{22,23}=6.8$ & & $17,21,23$ \\
\hline $\mathrm{C}-23$ & 36.0 & $23-\mathrm{CH}_{2}$ & 3.00 & & & $20,22,24$ \\
\hline $\mathrm{C}-24$ & 177.6 & & & & & \\
\hline $\mathrm{C}-1$, & 173.0 & & & & & \\
\hline $\mathrm{C}-2$ & 42.7 & $2^{\prime}-\mathrm{CH}_{2}$ & 2.40 & $\mathrm{~J}_{2^{\prime}, 3},=6$ & & $1^{\prime}, 3^{\prime}, 4^{\prime}$ \\
\hline $\mathrm{C}-3^{\prime}$ & 70.4 & $3^{\prime}-\mathrm{H}$ & 3.91 & $\mathrm{~J}_{3^{\prime}, 4^{\prime} \mathrm{a}}=6, \mathrm{~J}_{3^{\prime}, 4^{\prime} \mathrm{b}}=6$ & & \\
\hline \multirow[t]{2}{*}{ C-4' } & 30.6 & $4^{\prime}-\mathrm{H}_{\mathrm{a}}$ & 1.46 & $\mathrm{~J}_{4^{\prime} \mathrm{a}, \mathrm{y}^{\prime}}=7.4$ & & \\
\hline & & $4^{\prime}-\mathrm{H}_{\mathrm{b}}$ & 1.54 & $\mathrm{~J}_{4^{\prime} \mathrm{b}, 5^{\prime}}=7.4$ & & \\
\hline$C-5^{\prime}$ & 9.98 & $5^{\prime}-\mathrm{CH}_{3}$ & 0.95 & & & $4^{\prime}, 3^{\prime}$ \\
\hline C-1" & 172.3 & & & & & \\
\hline C-2" & 20.7 & 2"- $\mathrm{CH}_{3}$ & 2.09 & & & $1 "$ \\
\hline
\end{tabular}

$\mathrm{MS}\left(\mathrm{ESI}^{+}\right) \mathrm{m} / \mathrm{z}:(\mathrm{M}+\mathrm{H})^{+}$594.3, $(\mathrm{M}+\mathrm{Na})^{+} 616.3$ 


\section{Compound 2}

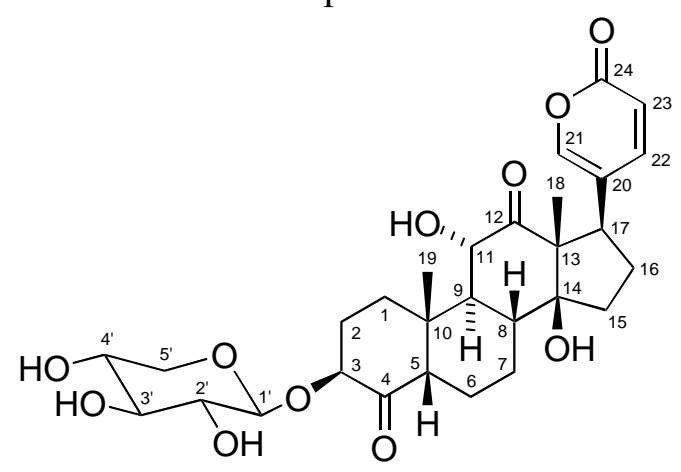

\begin{tabular}{|c|c|c|c|c|c|c|}
\hline $\begin{array}{c}\text { Carbon } \\
\text { No. }\end{array}$ & $\delta, \mathrm{ppm}$ & Proton No. & $\delta, \mathrm{ppm}$ & $J, \mathrm{~Hz}$ & $\begin{array}{c}\text { NOESY } \\
\text { Correlations }\end{array}$ & $\begin{array}{c}\text { HMBC } \\
\text { Correlations }\end{array}$ \\
\hline \multirow[t]{2}{*}{$\mathrm{C}-1$} & 32.6 & $1-\mathrm{H} \alpha$ & 2.58 & $\mathrm{~J}_{1 \alpha, 1 \beta}=13.5, \mathrm{~J}_{1 \alpha, 2 \alpha}=3$ & 11(w), 19 & \\
\hline & & $1-\mathrm{H} \beta$ & 2.02 & $\mathrm{~J}_{1 \beta, 2 \alpha}=13, \mathrm{~J}_{1 \beta, 2 \beta}=3$ & 5,19 & \\
\hline \multirow[t]{2}{*}{$\mathrm{C}-2$} & 28.6 & $2-\mathrm{H} \alpha$ & 2.13 & $\mathrm{~J}_{2 \alpha, 2 \beta}=13.5, \mathrm{~J}_{2 \alpha, 3}=2.9$ & & \\
\hline & & $2-\mathrm{H} \beta$ & 1.90 & $\mathrm{~J}_{2 \beta, 3}=2.9$ & & 4 \\
\hline $\mathrm{C}-3$ & 80.41 & $3-\mathrm{H}$ & 3.92 & & & $1^{\prime}, 4$ \\
\hline C-4 & 121.6 & & & & & \\
\hline$C-5$ & 54.3 & $5-\mathrm{H}$ & 2.95 & $\mathrm{~J}_{5,6 \alpha}=2-3, \mathrm{~J}_{5,6 \mathrm{~B}}=4-5$ & $6 \beta, 19$ & 4 \\
\hline \multirow[t]{2}{*}{$\mathrm{C}-6$} & 20.8 & 6-H $\alpha$ & 2.04 & $\mathrm{~J}_{6 \alpha, 6 \beta}=13, \mathrm{~J}_{6 \alpha, 7 \alpha}=4$ & & \\
\hline & & $6-\mathrm{H} \beta$ & 1.65 & $\mathrm{~J}_{6 \beta, 7 \alpha}=13, \mathrm{~J}_{6 \beta, 7 \beta}=4.5$ & 19 & 4 \\
\hline \multirow[t]{2}{*}{$\mathrm{C}-7$} & 24.1 & 7-H $\alpha$ & 1.51 & $\mathrm{~J}_{7 \alpha, 7 \beta}=13, \mathrm{~J}_{7 \alpha, 8}=13$ & & \\
\hline & & $7-\mathrm{H} \beta$ & 1.95 & $\mathrm{~J}_{7 \beta, 8}=3.4$ & & \\
\hline $\mathrm{C}-8$ & 39.9 & $8-\mathrm{H}$ & 2.14 & $\mathrm{~J}_{8,9}=11$ & 11,18 & \\
\hline C-9 & 44.8 & $9-\mathrm{H}$ & 1.41 & $\mathrm{~J}_{9,11}=11$ & & \\
\hline C-10 & 44.6 & & & & & \\
\hline C-11 & 74.3 & $11-\mathrm{H}$ & 4.37 & & 18,19 & 9,12 \\
\hline C-12 & 214.2 & & & & & \\
\hline C-13 & 63.4 & & & & & \\
\hline C-14 & 85.7 & & & & & \\
\hline \multirow[t]{2}{*}{$\mathrm{C}-15$} & 32.9 & $15-\mathrm{H}(\alpha$ or $\beta)$ & 1.70 & & & \\
\hline & & $15-\mathrm{H}(\alpha$ or $\beta)$ & 1.17 & & & \\
\hline \multirow[t]{2}{*}{$\mathrm{C}-16$} & 28.7 & $16-\mathrm{H}(\alpha$ or $\beta)$ & 1.70 & & & \\
\hline & & $16-\mathrm{H}(\alpha$ or $\beta)$ & 1.98 & & & \\
\hline C-17 & 41.8 & $17-\mathrm{H}$ & 4.11 & $\begin{array}{l}\mathrm{J}_{17,16(\alpha \text { or } \beta)}=6.9, \\
\mathrm{~J}_{17,16(\alpha \text { or } \beta)}=9.6\end{array}$ & $18(\mathrm{w}), 21,22$ & $\begin{array}{c}12,13,16,20, \\
21,22,\end{array}$ \\
\hline C-18 & 17.6 & $18-\mathrm{CH}_{3}$ & 0.90 & & & $12,13,14,17$ \\
\hline C-19 & 22.9 & $19-\mathrm{CH}_{3}$ & 1.36 & & & $1,5,9,10$ \\
\hline C-20 & 123.0 & & & & & \\
\hline C-21 & 151.6 & $21-\mathrm{H}$ & 7.51 & $\mathrm{~J}_{21,22}=2.6$ & & $20,22,24$ \\
\hline C-22 & 148.9 & $22-\mathrm{H}$ & 7.91 & $\mathrm{~J}_{22,23}=9.7$ & & 24,21 \\
\hline $\mathrm{C}-23$ & 115.6 & $23-\mathrm{H}$ & 6.30 & & & 20,24 \\
\hline $\mathrm{C}-24$ & 164.5 & & & & & \\
\hline $\mathrm{C}-1{ }^{\prime}$ & 103.0 & $1 '-H$ & 4.04 & $\mathrm{~J}_{1^{\prime}, 2^{\prime}}=7.6$ & 5,3 ', 5'ax & 3 \\
\hline $\mathrm{C}-2$ & 74.5 & $2^{\prime}-\mathrm{H}$ & 3.17 & $\mathbf{J}_{2,3},{ }^{\prime}=9.0$ & & \\
\hline $\mathrm{C}-3^{\prime}$ & 77.7 & 3'-H & 3.24 & $\mathrm{~J}_{3^{\prime}, 4^{\prime}}=8.8$ & & \\
\hline C-4' & 70.9 & 4'-H & 3.46 & $\mathrm{~J}_{4,5^{\prime} \mathrm{ax}}=11, \mathrm{~J}_{4,5 \mathrm{eq}}=5.4$ & & \\
\hline \multirow[t]{2}{*}{$\mathrm{C}-5$} & 66.9 & $5^{\prime}-\mathrm{H}_{\mathrm{ax}}$ & 3.12 & $\mathrm{~J}_{5^{\prime} \mathrm{ax}, \zeta^{\prime} \mathrm{eq}}=11$ & & \\
\hline & & $5^{\prime}-\mathrm{H}_{\mathrm{eq}}$ & 3.83 & & & \\
\hline
\end{tabular}




\section{Compound 3}

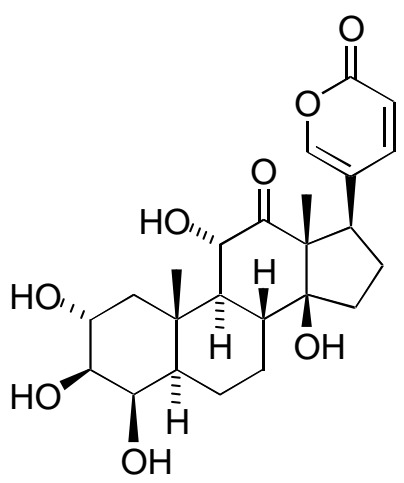

\begin{tabular}{|c|c|c|c|c|c|c|}
\hline $\begin{array}{c}\text { Carbon } \\
\text { No. }\end{array}$ & $\delta, \mathrm{ppm}$ & Proton No. & $\delta, \mathrm{ppm}$ & $J, \mathrm{~Hz}$ & $\begin{array}{c}\text { NOESY } \\
\text { Correlations }\end{array}$ & $\begin{array}{c}\text { HMBC } \\
\text { Correlations }\end{array}$ \\
\hline \multirow[t]{2}{*}{$\mathrm{C}-1$} & 48.5 & $1-\mathrm{H} \alpha$ & 1.13 & $\mathrm{~J}_{1 \alpha, 1 \beta}=13.5, \mathrm{~J}_{1 \alpha, 2}=11$ & 3 & \\
\hline & & $1-\mathrm{H} \beta$ & 2.83 & $\mathrm{~J}_{1 \beta, 2}=4.6$ & & $2,3,5,10,19$ \\
\hline $\mathrm{C}-2$ & 69.0 & 2-H & 3.84 & $\mathrm{~J}_{2,3}=9.6$ & 19 & \\
\hline C-3 & 78.0 & $3-\mathrm{H}$ & 3.25 & $\mathrm{~J}_{3,4}=3.7$ & & \\
\hline $\mathrm{C}-4$ & 76.4 & $4-\mathrm{H}$ & 3.82 & $\mathrm{~J}_{4,5}=3.5$ & & \\
\hline C-5 & 50.3 & $5-\mathrm{H}$ & 1.21 & $\mathrm{~J}_{5,6 \alpha}=3-4, \mathrm{~J}_{5,6 \beta}=13$ & & \\
\hline \multirow[t]{2}{*}{ C-6 } & 26.7 & 6-Ha & 1.51 & $\mathrm{~J}_{6 \alpha, 6 \beta}=13, \mathrm{~J}_{6 \alpha, 7 \alpha}=3-4$ & & \\
\hline & & $6-\mathrm{H} \beta$ & 1.82 & $J_{6 \beta, 7 \alpha}=13, J_{6 \beta, 7 \beta}=3-4$ & & \\
\hline \multirow[t]{2}{*}{$\mathrm{C}-7$} & 29.2 & $7-\mathrm{H \alpha}$ & 1.24 & $\mathrm{~J}_{7 \alpha, 7 \beta}=12.5, \mathrm{~J}_{7 \alpha, 8}=11.5$ & & \\
\hline & & $7-\mathrm{H} \beta$ & 2.28 & $\mathrm{~J}_{7 \beta, 8}=3.5$ & & \\
\hline $\mathrm{C}-8$ & 40.3 & $8-\mathrm{H}$ & 2.07 & $\mathrm{~J}_{8,9}=11.5$ & $11,18,19$ & \\
\hline C-9 & 55.0 & $9-\mathrm{H}$ & 1.15 & $\mathrm{~J}_{9,11}=11.3$ & & \\
\hline $\mathrm{C}-10$ & 40.3 & & & & & \\
\hline$C-11$ & 74.4 & $11-\mathrm{H}$ & 4.43 & & 18,19 & 9,12 \\
\hline $\mathrm{C}-12$ & 214.7 & & & & & \\
\hline $\mathrm{C}-13$ & 63.4 & & & & & \\
\hline C-14 & 85.7 & & & & & \\
\hline \multirow[t]{2}{*}{$\mathrm{C}-15$} & 33.1 & $15-\mathrm{H}(\alpha$ or $\beta)$ & 1.75 & & & \\
\hline & & $15-\mathrm{H}(\alpha$ or $\beta)$ & 1.28 & & & \\
\hline \multirow[t]{2}{*}{$\mathrm{C}-16$} & 28.9 & $16-\mathrm{H}(\alpha$ or $\beta)$ & 1.73 & & 21,22 & \\
\hline & & $16-\mathrm{H}(\alpha$ or $\beta)$ & 2.00 & & & \\
\hline $\mathrm{C}-17$ & 41.8 & $17-\mathrm{H}$ & 4.12 & $\begin{array}{c}\mathrm{J}_{17,16(\alpha \text { or } \beta)}=7.2, \\
\mathrm{~J}_{17,16(\alpha \text { or } \beta)}=9.5\end{array}$ & 18,22 & $\begin{array}{c}12,13,16,20 \\
21,22\end{array}$ \\
\hline C-18 & 17.9 & $18-\mathrm{CH}_{3}$ & 0.91 & & 21,22 & $12,13,14,17$ \\
\hline C-19 & 15.7 & $19-\mathrm{CH}_{3}$ & 1.32 & & & $1,5,9,10$ \\
\hline $\mathrm{C}-20$ & 123.1 & & & & & \\
\hline $\mathrm{C}-21$ & 151.4 & 21-H & 7.51 & $\mathrm{~J}_{21,22}=2.5$ & & $17,20,22,24$ \\
\hline $\mathrm{C}-22$ & 148.9 & 22-H & 7.91 & $\mathrm{~J}_{22,23}=9.7$ & & $17,21,24$ \\
\hline C-23 & 115.7 & $23-\mathrm{H}$ & 6.31 & & & 20,24 \\
\hline $\mathrm{C}-24$ & 164.2 & & & & & \\
\hline
\end{tabular}




\section{Compound $\mathbf{4}$}

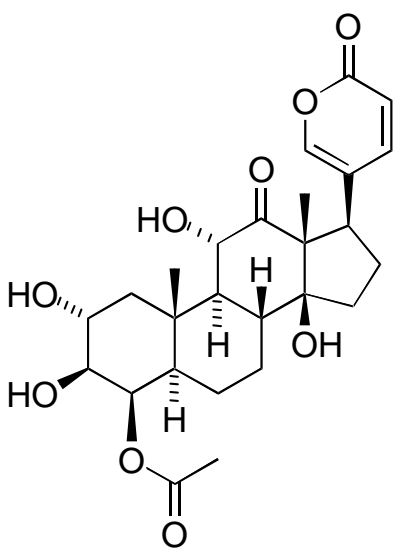

\begin{tabular}{|c|c|c|c|c|c|c|}
\hline $\begin{array}{l}\text { Carbon } \\
\text { No. }\end{array}$ & $\delta, \mathrm{ppm}$ & Proton No. & $\delta, \mathrm{ppm}$ & $J, \mathrm{~Hz}$ & $\begin{array}{c}\text { NOESY } \\
\text { Correlations }\end{array}$ & $\begin{array}{c}\text { HMBC } \\
\text { Correlations } \\
\end{array}$ \\
\hline \multirow{2}{*}{$\mathrm{C}-1$} & 48.2 & $1-\mathrm{H} \alpha$ & 1.20 & $\mathrm{~J}_{1 \alpha, 1 \mathrm{\beta}}=12.5, \mathrm{~J}_{1 \alpha, 2}=11.5$ & 3 & \\
\hline & & $1-\mathrm{H} \beta$ & 2.89 & $\mathrm{~J}_{1 \beta, 2}=4.4$ & & \\
\hline $\mathrm{C}-2$ & 69.0 & $2-\mathrm{H}$ & 3.82 & $\mathrm{~J}_{2,3}=10$ & 19 & \\
\hline $\mathrm{C}-3$ & 76.2 & $3-\mathrm{H}$ & 3.42 & $\mathrm{~J}_{3,4}=3.8$ & 5 & \\
\hline C-4 & 77.7 & 4-H & 5.30 & $\mathrm{~J}_{4,5}=2.0$ & & 1 ' \\
\hline C-5 & 49.5 & $5-\mathrm{H}$ & 1.49 & $\mathrm{~J}_{5,6 \alpha}=2-4, \mathrm{~J}_{5,6 \beta}=12.5$ & 7 & \\
\hline \multirow[t]{2}{*}{ C-6 } & 26.0 & 6-Ha & 1.51 & $\mathrm{~J}_{6 \alpha, 7 \alpha}=3$ & & \\
\hline & & $6-\mathrm{H} \beta$ & 1.46 & $\mathrm{~J}_{6 \beta, 7 \alpha}=12, \mathrm{~J}_{6 \beta, 7 \beta}=3$ & & \\
\hline \multirow[t]{2}{*}{$\mathrm{C}-7$} & 28.8 & $7-\mathrm{H \alpha}$ & 1.25 & $\mathrm{~J}_{7 \alpha, 7 \mathrm{p}}=12, \mathrm{~J}_{7 \alpha, 8}=12$ & & \\
\hline & & $7-\mathrm{H} \beta$ & 2.23 & $\mathrm{~J}_{7 \beta, 8}=3.5$ & & \\
\hline C-8 & 40.0 & $8-\mathrm{H}$ & 2.04 & $\mathrm{~J}_{8,9}=12$ & & \\
\hline C-9 & 54.8 & $9-\mathrm{H}$ & 1.19 & $\mathrm{~J}_{9,11}=11.2$ & 5 & \\
\hline $\mathrm{C}-10$ & 40.3 & & & & & \\
\hline $\mathrm{C}-11$ & 74.4 & $11-\mathrm{H}$ & 4.45 & & 18,19 & 12 \\
\hline C-12 & 214.6 & & & & & \\
\hline C-13 & 63.5 & & & & & \\
\hline $\mathrm{C}-14$ & 85.7 & & & & & \\
\hline \multirow[t]{2}{*}{$\mathrm{C}-15$} & 33.0 & $15-\mathrm{H}(\alpha$ or $\beta)$ & 1.75 & & & \\
\hline & & $15-\mathrm{H}(\alpha$ or $\beta)$ & 1.28 & & & \\
\hline \multirow[t]{2}{*}{$\mathrm{C}-16$} & 28.9 & $16-\mathrm{H}(\alpha$ or $\beta)$ & 1.72 & & & \\
\hline & & $16-\mathrm{H}(\alpha$ or $\beta)$ & 1.99 & & & \\
\hline $\mathrm{C}-17$ & 41.8 & $17-\mathrm{H}$ & 4.12 & $\begin{array}{l}\mathrm{J}_{17,16(\alpha \text { or } \beta)}=7.2, \\
\mathrm{~J}_{17,16(\alpha \text { or } \beta)}=9.8\end{array}$ & & $12,13,20,22$ \\
\hline C-18 & 17.9 & $18-\mathrm{CH}_{3}$ & 0.91 & & 8,11 & $12,13,14,17$ \\
\hline C-19 & 15.2 & $19-\mathrm{CH}_{3}$ & 1.29 & & $2,8,11$ & $1,5,9,10$ \\
\hline $\mathrm{C}-20$ & 123.0 & & & & & \\
\hline $\mathrm{C}-21$ & 151.4 & 21-H & 7.52 & $\mathrm{~J}_{21,22}=2.6$ & & $17,20,24$ \\
\hline $\mathrm{C}-22$ & 148.9 & 22-H & 7.91 & $\mathrm{~J}_{22,23}=9.7$ & & 17,24 \\
\hline $\mathrm{C}-23$ & 115.7 & $23-\mathrm{H}$ & 6.31 & & & 20,24 \\
\hline $\mathrm{C}-24$ & 164.3 & & & & & \\
\hline C-1' & 172.7 & & & & & \\
\hline C-2' & 21.0 & $2^{\prime}-\mathrm{CH}_{3}$ & 2.09 & & & $1^{\prime}$ \\
\hline
\end{tabular}




\section{Compound 5}

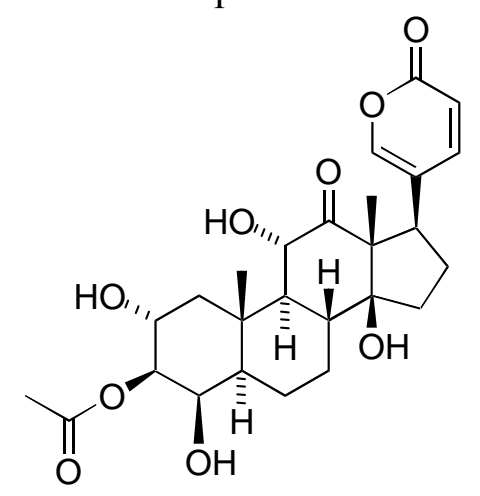

\begin{tabular}{|c|c|c|c|c|c|c|}
\hline $\begin{array}{l}\text { Carbon } \\
\text { No. }\end{array}$ & $\delta, \mathrm{ppm}$ & Proton No. & $\delta, \mathrm{ppm}$ & $J, \mathrm{~Hz}$ & $\begin{array}{c}\text { NOESY } \\
\text { Correlations }\end{array}$ & $\begin{array}{c}\text { HMBC } \\
\text { Correlations }\end{array}$ \\
\hline \multirow[t]{2}{*}{$\mathrm{C}-1$} & 48.7 & $1-\mathrm{H} \alpha$ & 1.22 & $\mathrm{~J}_{1 \alpha, 1 \mathrm{\beta}}=13.5, \mathrm{~J}_{1 \alpha, 2}=12$ & 3 & \\
\hline & & $1-\mathrm{H} \beta$ & 2.90 & $\mathrm{~J}_{1 \beta, 2}=4.5$ & 19 & $2,3,5,10$ \\
\hline $\mathrm{C}-2$ & 66.2 & $2-\mathrm{H}$ & 4.07 & $\mathrm{~J}_{2,3}=10.2$ & 19 & \\
\hline $\mathrm{C}-3$ & 81.0 & $3-\mathrm{H}$ & 4.51 & $\mathrm{~J}_{3,4}=3.6$ & 5 & 1 ' \\
\hline C-4 & 73.7 & $4-\mathrm{H}$ & 3.96 & $\mathrm{~J}_{4,5}=3-4$ & $6 \alpha$ & \\
\hline$C-5$ & 50.0 & $5-\mathrm{H}$ & 1.28 & $\mathrm{~J}_{5,6 \mathrm{\alpha}}=2-3, \mathrm{~J}_{5,6 \beta}=12.5$ & & \\
\hline \multirow[t]{2}{*}{ C-6 } & 26.5 & 6-H $\alpha$ & 1.47 & $\mathrm{~J}_{6 \alpha, 6 \beta}=12.5, \mathrm{~J}_{6 \alpha, 7 \alpha}=3$ & & \\
\hline & & $6-\mathrm{H} \beta$ & 1.81 & $\mathrm{~J}_{6 \beta, 7 \alpha}=12.5, \mathrm{~J}_{6 \beta, 7 \beta}=3-4$ & & \\
\hline \multirow[t]{2}{*}{$\mathrm{C}-7$} & 29.1 & 7-H $\alpha$ & 1.26 & $\mathrm{~J}_{7 \alpha, 7 \mathrm{\beta}}=13, \mathrm{~J}_{7 \alpha, 8}=12$ & & \\
\hline & & 7-H $\beta$ & 2.28 & $\mathrm{~J}_{7 \beta, 8}=3-4$ & & \\
\hline $\mathrm{C}-8$ & 40.2 & $8-\mathrm{H}$ & 2.06 & $\mathrm{~J}_{8,9}=12$ & $11,18,19$ & \\
\hline C-9 & 54.9 & $9-\mathrm{H}$ & 1.18 & $\mathrm{~J}_{9,11}=11.6$ & & \\
\hline $\mathrm{C}-10$ & 40.3 & & & & & \\
\hline $\mathrm{C}-11$ & 74.4 & $11-\mathrm{H}$ & 4.44 & & 18,19 & 9,12 \\
\hline $\mathrm{C}-12$ & 214.8 & & & & & \\
\hline C-13 & 63.5 & & & & & \\
\hline $\mathrm{C}-14$ & 86.1 & & & & & \\
\hline \multirow[t]{2}{*}{$\mathrm{C}-15$} & 33.1 & $15-\mathrm{H}(\alpha$ or $\beta)$ & 1.72 & & & \\
\hline & & $15-\mathrm{H}(\alpha$ or $\beta)$ & 1.29 & & & \\
\hline \multirow[t]{2}{*}{$\mathrm{C}-16$} & 28.9 & $16-\mathrm{H}(\alpha$ or $\beta)$ & 1.72 & & 22 & \\
\hline & & $16-\mathrm{H}(\alpha$ or $\beta)$ & 1.99 & & 17 & \\
\hline $\mathrm{C}-17$ & 41.8 & $17-\mathrm{H}$ & 4.11 & $\begin{array}{l}\mathrm{J}_{17,16(\alpha \text { or } \beta)}=7.3, \\
\mathrm{~J}_{17,16(\alpha \text { or } \beta)}=9.6\end{array}$ & $18(\mathrm{w}), 21$ & $\begin{array}{c}12,13,16,20, \\
21,22,\end{array}$ \\
\hline $\mathrm{C}-18$ & 17.9 & $18-\mathrm{CH}_{3}$ & 0.90 & & 22 & $12,13,14,17$ \\
\hline $\mathrm{C}-19$ & 15.6 & $19-\mathrm{CH}_{3}$ & 1.32 & & & $1,5,9,10$ \\
\hline $\mathrm{C}-20$ & 123.3 & & & & & \\
\hline $\mathrm{C}-21$ & 151.4 & 21-H & 7.51 & $\mathrm{~J}_{21,22}=2.6$ & & $20,22,24$ \\
\hline $\mathrm{C}-22$ & 149.0 & $22-\mathrm{H}$ & 7.91 & $\mathrm{~J}_{22,23}=9.7$ & & 21,24 \\
\hline $\mathrm{C}-23$ & 115.8 & $23-\mathrm{H}$ & 6.31 & & & 20,24 \\
\hline $\mathrm{C}-24$ & 164.5 & & & & & \\
\hline $\mathrm{C}-1^{\prime}$ & 173.0 & & & & & \\
\hline $\mathrm{C}-2$ & 21.1 & 2'- $-\mathrm{CH}_{3}$ & 2.10 & & & 1 ' \\
\hline
\end{tabular}




\section{Compound 6}

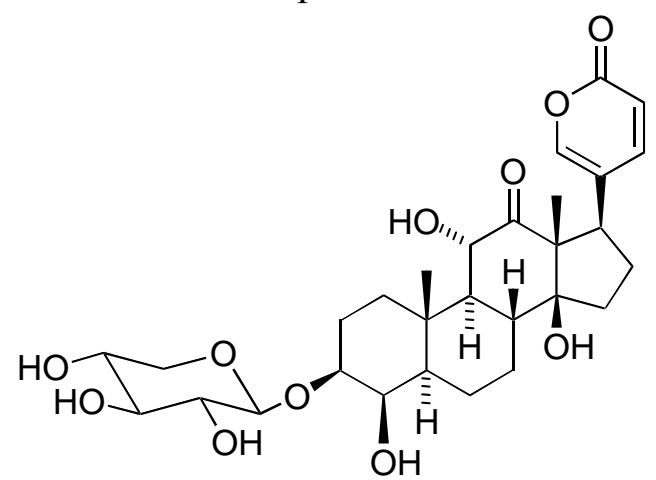

\begin{tabular}{|c|c|c|c|c|c|c|}
\hline $\begin{array}{l}\text { Carbon } \\
\text { No. }\end{array}$ & $\delta, \mathrm{ppm}$ & Proton No. & $\delta, \mathrm{ppm}$ & $J, \mathrm{~Hz}$ & $\begin{array}{c}\text { NOESY } \\
\text { Correlations }\end{array}$ & $\begin{array}{c}\text { HMBC } \\
\text { Correlations }\end{array}$ \\
\hline \multirow[t]{2}{*}{$\mathrm{C}-1$} & 39.8 & $1-\mathrm{H} \alpha$ & 1.16 & $\mathrm{~J}_{1 \alpha, 1 \beta}=13, \mathrm{~J}_{1 \alpha, 2 \alpha}=3.5$ & 3 & \\
\hline & & $1-\mathrm{H} \beta$ & 2.54 & $\mathrm{~J}_{1 \beta, 2 \alpha}=3.5, \mathrm{~J}_{1 \beta, 2 \beta}=3.5$ & 19 & \\
\hline \multirow[t]{2}{*}{$\mathrm{C}-2$} & 24.9 & $2-\mathrm{H \alpha}$ & 1.58 & $\mathrm{~J}_{2 \alpha, 2 \beta}=13, \mathrm{~J}_{2 \alpha, 3}=4$ & & \\
\hline & & $2-\mathrm{H} \beta$ & 1.89 & $\mathrm{~J}_{2 \beta, 1 \alpha}=13, \mathrm{~J}_{2 \beta, 3}=12$ & 19 & \\
\hline $\mathrm{C}-3$ & 80.3 & $3-\mathrm{H}$ & 3.62 & $\mathrm{~J}_{3,4}=2-3$ & 5 & 1 , \\
\hline C-4 & 72.9 & $4-\mathrm{H}$ & 3.9 & $\mathrm{~J}_{4,5}=2-3$ & 5 & \\
\hline $\mathrm{C}-5$ & 49.7 & $5-\mathrm{H}$ & 1.15 & $\mathrm{~J}_{5,6 \alpha}=3, \mathrm{~J}_{5,6 \beta}=13$ & 9 & \\
\hline \multirow[t]{2}{*}{ C-6 } & 27.3 & $6-\mathrm{H} \alpha$ & 1.47 & $\mathrm{~J}_{6 \alpha, 6 \beta}=13, \mathrm{~J}_{6 \alpha, 7 \alpha}=3.5$ & & \\
\hline & & $6-\mathrm{H} \beta$ & 1.86 & $\mathrm{~J}_{6 \beta, 7 \alpha}=13, \mathrm{~J}_{6 \beta, 7 \beta}=3.6$ & 8,19 & 5,7 \\
\hline \multirow[t]{2}{*}{$\mathrm{C}-7$} & 29.2 & 7-H $\alpha$ & 1.22 & $\mathrm{~J}_{7 \alpha, 7 \beta}=13, \mathrm{~J}_{7 \alpha, 8}=12$ & & \\
\hline & & 7-H $\beta$ & 2.28 & $\mathrm{~J}_{7 \beta, 6 \alpha}=2-3, \mathrm{~J}_{7 \beta, 8}=3.7$ & & \\
\hline C-8 & 40.7 & $8-\mathrm{H}$ & 2.10 & $\mathrm{~J}_{8,9}=12$ & 19 & \\
\hline C-9 & 55.0 & $9-\mathrm{H}$ & 1.10 & $\mathrm{~J}_{9,11}=11.3$ & & \\
\hline $\mathrm{C}-10$ & 38.8 & & & & & \\
\hline $\mathrm{C}-11$ & 74.3 & $11-\mathrm{H}$ & 4.42 & & 19,18 & $9,10,12$ \\
\hline $\mathrm{C}-12$ & 214.7 & & & & & \\
\hline $\mathrm{C}-13$ & 63.5 & & & & & \\
\hline C-14 & 85.7 & & & & & \\
\hline \multirow[t]{2}{*}{$\mathrm{C}-15$} & 32.9 & $15-\mathrm{H}(\alpha$ or $\beta)$ & 1.75 & & & \\
\hline & & $15-\mathrm{H}(\alpha$ or $\beta)$ & 1.29 & & & \\
\hline \multirow[t]{2}{*}{$\mathrm{C}-16$} & 28.8 & $16-\mathrm{H}(\alpha$ or $\beta)$ & 1.73 & & 22 & 20 \\
\hline & & $16-\mathrm{H}(\alpha$ or $\beta)$ & 2.00 & & & \\
\hline $\mathrm{C}-17$ & 41.8 & $17-\mathrm{H}$ & 4.13 & $\begin{array}{l}\mathrm{J}_{17,16(\alpha \text { or } \beta)}=6.9, \\
\mathrm{~J}_{17,16(\alpha \text { or } \beta)}=9.7\end{array}$ & & $\begin{array}{c}12,13,16,20 \\
21,22\end{array}$ \\
\hline C-18 & 17.8 & $18-\mathrm{CH}_{3}$ & 0.91 & & 22 & $12,13,14,17$ \\
\hline C-19 & 14.7 & $19-\mathrm{CH}_{3}$ & 1.28 & & & $1,5,9,10$ \\
\hline $\mathrm{C}-20$ & 122.9 & & & & & \\
\hline $\mathrm{C}-21$ & 151.3 & 21-H & 7.51 & $\mathrm{~J}_{21,22}=2.6$ & & $17,20,22,24$ \\
\hline $\mathrm{C}-22$ & 148.9 & $22-\mathrm{H}$ & 7.92 & $\mathrm{~J}_{22,23}=9.6$ & & $17,21,24$ \\
\hline $\mathrm{C}-23$ & 115.6 & $23-\mathrm{H}$ & 6.31 & & & 20,24 \\
\hline $\mathrm{C}-24$ & 164.3 & & & & & \\
\hline $\mathrm{C}-1$ & 102.6 & $1 '-H$ & 4.36 & $\mathrm{~J}_{1^{\prime}, 2^{\prime}}=7.6$ & $3,4,3^{\prime}, 5^{\prime}$ 'ax & 3 \\
\hline C-2, & 74.9 & $2^{\prime}-\mathrm{H}$ & 3.18 & $\mathrm{~J}_{2}{ }^{\prime},{ }^{3}=9.0$ & & \\
\hline C-3' & 77.7 & $3^{\prime}-\mathrm{H}$ & 3.32 & $\mathrm{~J}_{3,4},=8.8$ & & \\
\hline C-4' & 80.0 & $4 '-\mathrm{H}$ & 3.48 & $\mathrm{~J}_{4,5 \mathrm{ax}}=11, \mathrm{~J}_{4,5 \mathrm{seq}}=5.4$ & & \\
\hline \multirow[t]{2}{*}{$\mathrm{C}-5$} & 66.9 & $5^{\prime}-\mathrm{H}_{\mathrm{ax}}$ & 3.19 & 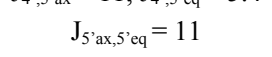 & & \\
\hline & & $5^{\prime}-\mathrm{H}_{\mathrm{eq}}$ & 3.83 & & & \\
\hline
\end{tabular}

MS $\left(\mathrm{ESI}^{+}\right) \mathrm{m} / \mathrm{z}:(\mathrm{M}+\mathrm{H})^{+}$565.3, $(\mathrm{M}+\mathrm{Na})^{+} 587.3$ 


\section{Compound 7}

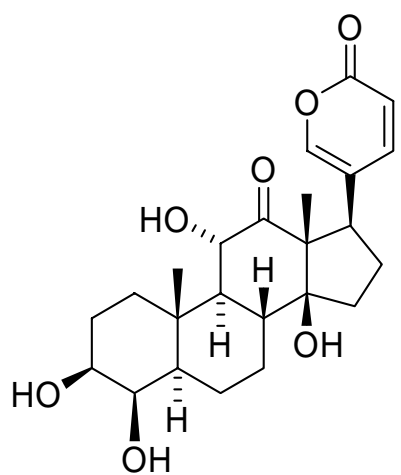

\begin{tabular}{|c|c|c|c|c|c|c|}
\hline $\begin{array}{c}\text { Carbon } \\
\text { No. }\end{array}$ & $\delta, \mathrm{ppm}$ & Proton No. & $\delta, \mathrm{ppm}$ & $J, \mathrm{~Hz}$ & $\begin{array}{c}\text { NOESY } \\
\text { Correlations }\end{array}$ & $\begin{array}{c}\text { HMBC } \\
\text { Correlations }\end{array}$ \\
\hline \multirow[t]{2}{*}{$\mathrm{C}-1$} & 39.8 & $1-\mathrm{H} \alpha$ & 1.15 & $\mathrm{~J}_{1 \alpha, 1 \beta}=13, \mathrm{~J}_{1 \alpha, 2 \alpha}=3.5$ & 3 & \\
\hline & & $1-\mathrm{H} \beta$ & 2.51 & $\mathrm{~J}_{1 \beta, 2 \alpha}=3.5, \mathrm{~J}_{1 \beta, 2 \beta}=3.5$ & & 10 \\
\hline \multirow[t]{2}{*}{$\mathrm{C}-2$} & 26.4 & $2-\mathrm{H} \alpha$ & 1.52 & $\mathrm{~J}_{2 \alpha, 2 \beta}=13, \mathrm{~J}_{2 \alpha, 3}=3.5$ & & \\
\hline & & $2-\mathrm{H} \beta$ & 1.79 & $\mathrm{~J}_{2 \beta, 1 \alpha}=13, \mathrm{~J}_{2 \beta, 3}=13$ & 19 & \\
\hline C-3 & 73.0 & $3-\mathrm{H}$ & 3.47 & $\mathrm{~J}_{3,4}=2.5$ & 5 & \\
\hline C-4 & 75.8 & $4-\mathrm{H}$ & 3.70 & $\mathrm{~J}_{4,5}=2.5$ & & \\
\hline $\mathrm{C}-5$ & 50.3 & $5-\mathrm{H}$ & 1.13 & $\mathrm{~J}_{5,6 \mathrm{\alpha}}=3, \mathrm{~J}_{5,6 \mathrm{\beta}}=13$ & & \\
\hline \multirow[t]{2}{*}{ C- 6} & 27.3 & 6-H $\alpha$ & 1.45 & $\mathrm{~J}_{6 \alpha, 6 \beta}=13, \mathrm{~J}_{6 \alpha, 7 \alpha}=3.5$ & & \\
\hline & & $6-\mathrm{H} \beta$ & 1.85 & $\mathrm{~J}_{\sigma \beta, 7 \alpha}=13, \mathrm{~J}_{\sigma \beta, 7 \beta}=3.6$ & 8 & \\
\hline \multirow{2}{*}{$\mathrm{C}-7$} & 29.2 & 7-H $\alpha$ & 1.22 & $\mathrm{~J}_{7 \alpha, 7 \mathrm{p}}=13, \mathrm{~J}_{7 \alpha, 8}=12$ & & \\
\hline & & $7-\mathrm{H} \beta$ & 2.27 & $\mathrm{~J}_{7 \beta, 6 \alpha}=2-4, \mathrm{~J}_{7 \beta, 8}=3.7$ & & \\
\hline C- 8 & 40.7 & $8-\mathrm{H}$ & 2.08 & $\mathrm{~J}_{8,9}=12$ & 11 & \\
\hline C-9 & 55.1 & $9-\mathrm{H}$ & 1.07 & $\mathrm{~J}_{9,11}=11.3$ & & \\
\hline $\mathrm{C}-10$ & 39.0 & & & & & \\
\hline $\mathrm{C}-11$ & 74.4 & $11-\mathrm{H}$ & 4.42 & & 18,19 & $9,10,12$ \\
\hline $\mathrm{C}-12$ & 214.9 & & & & & \\
\hline C-13 & 63.7 & & & & & \\
\hline C-14 & 85.9 & & & & & \\
\hline \multirow[t]{2}{*}{$C-15$} & 33.0 & $15-\mathrm{H}(\alpha$ or $\beta)$ & 1.71 & & & \\
\hline & & $15-\mathrm{H}(\alpha$ or $\beta)$ & 1.26 & & & \\
\hline \multirow[t]{2}{*}{ C-16 } & 28.8 & $16-\mathrm{H}(\alpha$ or $\beta)$ & 1.71 & & 21,22 & 20 \\
\hline & & $16-\mathrm{H}(\alpha$ or $\beta)$ & 1.98 & & & \\
\hline $\mathrm{C}-17$ & 41.7 & $17-\mathrm{H}$ & 4.11 & $\begin{aligned} \mathrm{J}_{17,16(\alpha \text { or } \beta} & =6.9, \\
\mathrm{~J}_{17,16(\alpha \text { or } \beta)} & =9.7\end{aligned}$ & $18(\mathrm{w}), 21,22$ & $\begin{array}{c}12,13,16,20, \\
21,22\end{array}$ \\
\hline C-18 & 17.8 & $18-\mathrm{CH}_{3}$ & 0.89 & & 21,22 & $12,13,14,17$ \\
\hline C-19 & 14.8 & 19- $\mathrm{CH}_{3}$ & 1.25 & & & $1,5,9,10$ \\
\hline $\mathrm{C}-20$ & 123.2 & & & & & \\
\hline $\mathrm{C}-21$ & 151.3 & 21-H & 7.51 & $\mathrm{~J}_{21,22}=2.6$ & & $17,20,22,24$ \\
\hline $\mathrm{C}-22$ & 149.0 & $22-\mathrm{H}$ & 7.91 & $\mathrm{~J}_{22,23}=9.6$ & & 21,24 \\
\hline $\mathrm{C}-23$ & 115.7 & $23-\mathrm{H}$ & 6.31 & & & 20,24 \\
\hline $\mathrm{C}-24$ & 164.3 & & & & & \\
\hline
\end{tabular}




\section{Compound 8}

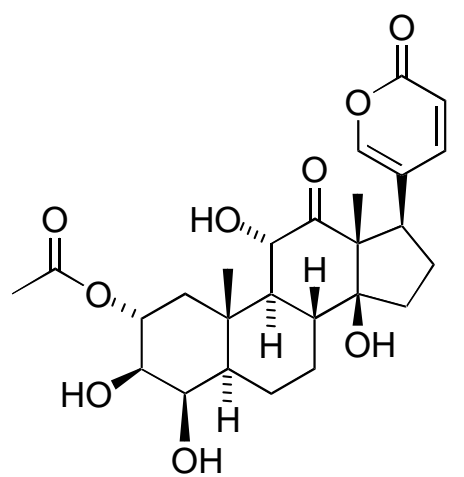

\begin{tabular}{|c|c|c|c|c|c|c|}
\hline $\begin{array}{l}\text { Carbon } \\
\text { No. }\end{array}$ & $\delta, \mathrm{ppm}$ & Proton No. & $\delta, \mathrm{ppm}$ & $J, \mathrm{~Hz}$ & $\begin{array}{c}\text { NOESY } \\
\text { Correlations }\end{array}$ & $\begin{array}{c}\text { HMBC } \\
\text { Correlations } \\
\end{array}$ \\
\hline \multirow[t]{2}{*}{$\mathrm{C}-1$} & 45.3 & $1-\mathrm{H} \alpha$ & 1.19 & $\mathrm{~J}_{1 \alpha, 1 \beta}=12, \mathrm{~J}_{1 \alpha, 2}=11.7$ & & \\
\hline & & $1-\mathrm{H} \beta$ & 2.80 & $\mathrm{~J}_{1 \beta, 2}=4.4$ & 19 & \\
\hline $\mathrm{C}-2$ & 73.4 & $2-\mathrm{H}$ & 5.17 & $\mathrm{~J}_{2,3}=10.1$ & 19 & 1 ' \\
\hline $\mathrm{C}-3$ & 75.1 & $3-\mathrm{H}$ & 3.49 & $\mathrm{~J}_{3,4}=3.8$ & 1,5 & 2 \\
\hline C-4 & 76.2 & $4-\mathrm{H}$ & 3.87 & $\mathrm{~J}_{4,5}=4.5$ & & \\
\hline C-5 & 50.2 & $5-\mathrm{H}$ & 1.26 & $\mathrm{~J}_{5,6 \alpha}=2-3, \mathrm{~J}_{5,6 \beta}=12.5$ & & \\
\hline \multirow[t]{2}{*}{ C-6 } & 26.6 & 6-Ha & 1.52 & $\mathrm{~J}_{6 \alpha, 6 \beta}=12.5, \mathrm{~J}_{6 \alpha, 7 \alpha}=2-3$ & & \\
\hline & & $6-\mathrm{H} \beta$ & 1.84 & $\mathrm{~J}_{6 \beta, 7 \alpha}=12.5, \mathrm{~J}_{6 \beta, 7 \beta}=3.6$ & 19 & \\
\hline \multirow[t]{2}{*}{$\mathrm{C}-7$} & 29.2 & 7-H $\alpha$ & 1.25 & $\mathrm{~J}_{7 \alpha, 7 \mathrm{\beta}}=12.5, \mathrm{~J}_{7 \alpha, 8}=12$ & & \\
\hline & & $7-\mathrm{H} \beta$ & 2.28 & $\mathrm{~J}_{7 \beta, 8}=3$ & & \\
\hline C-8 & 40.1 & $8-\mathrm{H}$ & 2.07 & $\mathrm{~J}_{8,9}=12$ & $11,18,19$ & \\
\hline C-9 & 54.9 & 9-H & 1.16 & $\mathrm{~J}_{9,11}=11.2$ & & \\
\hline $\mathrm{C}-10$ & 40.3 & & & & & \\
\hline C-11 & 74.2 & $11-\mathrm{H}$ & 4.42 & & 18,19 & $9,10,12$ \\
\hline $\mathrm{C}-12$ & 214.6 & & & & & \\
\hline $\mathrm{C}-13$ & 63.3 & & & & & \\
\hline C-14 & 85.7 & & & & & \\
\hline \multirow[t]{2}{*}{$\mathrm{C}-15$} & 33.0 & $15-\mathrm{H}(\alpha$ or $\beta)$ & 1.75 & & & \\
\hline & & $15-\mathrm{H}(\alpha$ or $\beta)$ & 1.26 & & & \\
\hline \multirow[t]{2}{*}{$\mathrm{C}-16$} & 28.8 & $16-\mathrm{H}(\alpha$ or $\beta)$ & 1.71 & & 22 & \\
\hline & & $16-\mathrm{H}(\alpha$ or $\beta)$ & 1.99 & & & \\
\hline $\mathrm{C}-17$ & 41.7 & $17-\mathrm{H}$ & 4.12 & $\begin{array}{l}J_{17,16(\alpha \text { or } \beta)}=7.1, \\
J_{17,16(\alpha \text { or } \beta)}=9.6\end{array}$ & $18(w), 21$ & $\begin{array}{c}12,13,16,20 \\
21,22\end{array}$ \\
\hline $\mathrm{C}-18$ & 17.9 & $18-\mathrm{CH}_{3}$ & 0.91 & & 22 & $12,13,14,17$ \\
\hline C-19 & 15.5 & $19-\mathrm{CH}_{3}$ & 1.36 & & & $1,5,9,10$ \\
\hline $\mathrm{C}-20$ & 122.9 & & & & & \\
\hline $\mathrm{C}-21$ & 151.4 & 21-H & 7.51 & $\mathrm{~J}_{21,22}=2.5$ & & $17,20,22,24$ \\
\hline $\mathrm{C}-22$ & 149.0 & $22-\mathrm{H}$ & 7.91 & $\mathrm{~J}_{22,23}=9.7$ & & 21,24 \\
\hline $\mathrm{C}-23$ & 115.7 & $23-\mathrm{H}$ & 6.31 & & & 20,24 \\
\hline $\mathrm{C}-24$ & 164.3 & & & & & \\
\hline $\mathrm{C}-1$ & 172.8 & & & & & \\
\hline C-2' & 21.2 & $2^{\prime}-\mathrm{CH}_{3}$ & 2.04 & & & 1 ' \\
\hline
\end{tabular}




\section{Compound 9}

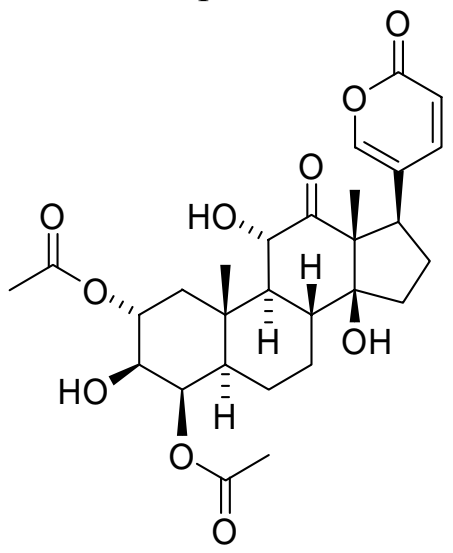

\begin{tabular}{|c|c|c|c|c|c|c|}
\hline $\begin{array}{c}\text { Carbon } \\
\text { No. }\end{array}$ & $\delta, \mathrm{ppm}$ & Proton No. & $\delta, \mathrm{ppm}$ & $J, \mathrm{~Hz}$ & $\begin{array}{c}\text { NOESY } \\
\text { Correlations }\end{array}$ & $\begin{array}{c}\text { HMBC } \\
\text { Correlations }\end{array}$ \\
\hline \multirow[t]{2}{*}{$\mathrm{C}-1$} & 44.9 & $1-\mathrm{H} \alpha$ & 1.26 & $\mathrm{~J}_{1 \alpha, 1 \beta}=13.2, \mathrm{~J}_{1 \alpha, 2}=11.7$ & 3 & \\
\hline & & $1-\mathrm{H} \beta$ & 2.87 & $\mathrm{~J}_{1 \beta, 2}=4.4$ & & \\
\hline $\mathrm{C}-2$ & 72.9 & $2-\mathrm{H}$ & 5.13 & $\mathrm{~J}_{2,3}=10.4$ & 19 & 1 ' \\
\hline $\mathrm{C}-3$ & 73.1 & $3-\mathrm{H}$ & 3.68 & $\mathrm{~J}_{3,4}=4.0$ & 5 & \\
\hline C-4 & 77.3 & $4-\mathrm{H}$ & 5.34 & $\mathrm{~J}_{4,5}=2.5$ & & $1 "$ \\
\hline$C-5$ & 48.9 & $5-\mathrm{H}$ & 1.54 & $\mathrm{~J}_{5,6 \alpha}=2-3, \mathrm{~J}_{5,6 \mathrm{\beta}}=12$ & & \\
\hline \multirow[t]{2}{*}{ C-6 } & 25.8 & $6-\mathrm{H \alpha}$ & 1.53 & $\mathrm{~J}_{6 \alpha, 6 \beta}=12.5, \mathrm{~J}_{6 \alpha, 7 \alpha}=3-4$ & & \\
\hline & & $6-\mathrm{H} \beta$ & 1.48 & $\mathrm{~J}_{6 \beta, 7 \alpha}=12.5, \mathrm{~J}_{\sigma \beta, 7 \beta}=3$ & & \\
\hline \multirow[t]{2}{*}{$\mathrm{C}-7$} & 28.6 & 7-H $\alpha$ & 1.25 & $\mathbf{J}_{7 \alpha, 7 \beta}=13, \mathbf{J}_{7 \alpha, 8}=12$ & & \\
\hline & & 7-H $\beta$ & 2.23 & $\mathrm{~J}_{7 \beta, 8}=3$ & & \\
\hline$C-8$ & 39.8 & $8-\mathrm{H}$ & 2.04 & $\mathrm{~J}_{8,9}=12$ & $11,18,19$ & \\
\hline C-9 & 54.5 & 9-H & 1.20 & $\mathrm{~J}_{9,11}=11.3$ & & \\
\hline $\mathrm{C}-10$ & 40.1 & & & & & \\
\hline$C-11$ & 74.1 & $11-\mathrm{H}$ & 4.44 & & 18,19 & 9,12 \\
\hline $\mathrm{C}-12$ & 214.3 & & & & & \\
\hline $\mathrm{C}-13$ & 63.3 & & & & & \\
\hline C-14 & 85.5 & & & & & \\
\hline \multirow[t]{2}{*}{$C-15$} & 33.0 & $15-\mathrm{H}(\alpha$ or $\beta)$ & 1.74 & & & \\
\hline & & $15-\mathrm{H}(\alpha$ or $\beta)$ & 1.27 & & & \\
\hline \multirow[t]{2}{*}{$C-16$} & 28.7 & $16-\mathrm{H}(\alpha$ or $\beta)$ & 1.71 & & 22 & \\
\hline & & $16-\mathrm{H}(\alpha$ or $\beta)$ & 1.99 & & & \\
\hline $\mathrm{C}-17$ & 41.7 & $17-\mathrm{H}$ & 4.11 & $\begin{array}{l}\mathrm{J}_{17,16(\alpha \text { or } \beta)}=7.1, \\
\mathrm{~J}_{17,16(\alpha \text { or } \beta)}=9.5\end{array}$ & $18(w), 21$ & $\begin{array}{c}12,13,16,20 \\
21,22\end{array}$ \\
\hline C-18 & 17.8 & $18-\mathrm{CH}_{3}$ & 0.91 & & 22 & $12,13,14,17$ \\
\hline C-19 & 14.9 & $19-\mathrm{CH}_{3}$ & 1.33 & & & $1,5,9,10$ \\
\hline $\mathrm{C}-20$ & 123.9 & & & & & \\
\hline C-21 & 151.4 & 21-H & 7.51 & $\mathrm{~J}_{21,22}=2.6$ & & $20,22,24$ \\
\hline $\mathrm{C}-22$ & 148.9 & $22-\mathrm{H}$ & 7.91 & $\mathrm{~J}_{22,23}=9.6$ & & 21,24 \\
\hline $\mathrm{C}-23$ & 115.7 & $23-\mathrm{H}$ & 6.31 & & & 20,24 \\
\hline $\mathrm{C}-24$ & 164.2 & & & & & \\
\hline \multicolumn{7}{|l|}{ C-2 Ac: } \\
\hline $\mathrm{C}-1$ & 171.1 & & & & & \\
\hline $\mathrm{C}-2^{\prime}$ & 19.7 & $2^{\prime}-\mathrm{CH}_{3}$ & 2.03 & & & 1 ' \\
\hline \multicolumn{7}{|l|}{ C-4 Ac: } \\
\hline $\mathrm{C}-1 "$ & 171.0 & & & & & \\
\hline C-2” & 19.6 & $2 "-\mathrm{CH}_{3}$ & 2.12 & & & $1 "$ \\
\hline
\end{tabular}




\section{Compound 10}

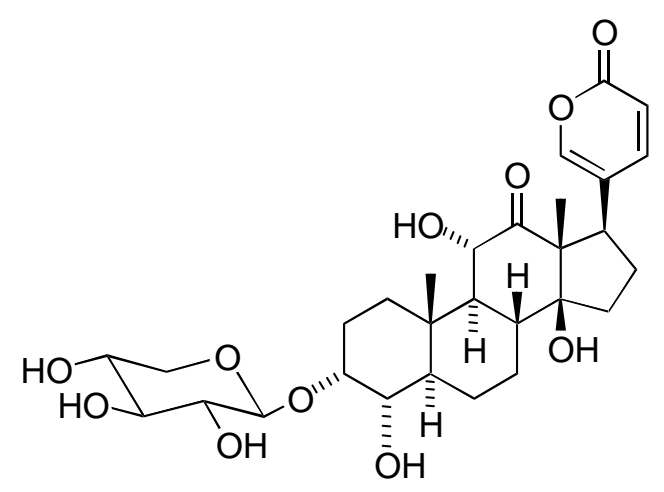

\begin{tabular}{|c|c|c|c|c|c|c|}
\hline $\begin{array}{l}\text { Carbon } \\
\text { No. }\end{array}$ & $\delta, \mathrm{ppm}$ & Proton No. & $\delta, \mathrm{ppm}$ & $J, \mathrm{~Hz}$ & $\begin{array}{c}\text { NOESY } \\
\text { Correlations }\end{array}$ & $\begin{array}{c}\text { HMBC } \\
\text { Correlations }\end{array}$ \\
\hline \multirow[t]{2}{*}{$\mathrm{C}-1$} & 32.9 & $1-\mathrm{H} \alpha$ & 1.44 & $\mathrm{~J}_{1 \alpha, 1 \beta}=13.6, \mathrm{~J}_{1 \alpha, 2 \alpha}=3-4$ & & \\
\hline & & $1-\mathrm{H} \beta$ & 2.42 & $\mathrm{~J}_{1 \beta, 2 \alpha}=3.2, \mathrm{~J}_{1 \beta, 2 \beta}=3.2$ & 19 & \\
\hline \multirow[t]{2}{*}{$\mathrm{C}-2$} & 27.3 & $2-\mathrm{H \alpha}$ & 1.83 & $\mathrm{~J}_{2 \alpha, 2 \beta}=14, \mathrm{~J}_{2 \alpha, 3}=3.2$ & & \\
\hline & & $2-\mathrm{H} \beta$ & 1.71 & $\mathrm{~J}_{2 \beta, 1 \alpha}=14, \mathrm{~J}_{2 \beta, 3}=3-4$ & & \\
\hline $\mathrm{C}-3$ & 82.9 & $3-\mathrm{H}$ & 3.90 & $\mathrm{~J}_{3,4}=3.2$ & & \\
\hline C-4 & 69.2 & $4-\mathrm{H}$ & 3.74 & $\mathrm{~J}_{4,5}=11$ & & \\
\hline$C-5$ & 46.9 & $5-\mathrm{H}$ & 1.70 & $\mathrm{~J}_{5,6 \alpha}=2-4, \mathrm{~J}_{5,6 \beta}=?$ & & \\
\hline \multirow[t]{2}{*}{ C-6 } & 21.2 & $6-\mathrm{H} \alpha$ & 2.04 & $\mathrm{~J}_{6 \alpha, 6 \beta}=13, \mathrm{~J}_{6 \alpha, 7 \alpha}=?$ & & \\
\hline & & $6-\mathrm{H} \beta$ & 1.69 & $\mathrm{~J}_{6 \beta, 7 \alpha}=13, \mathrm{~J}_{6 \beta, 7 \beta}=?$ & & \\
\hline \multirow[t]{2}{*}{$\mathrm{C}-7$} & 22.0 & 7-H $\alpha$ & 1.38 & $\mathrm{~J}_{\mathrm{J}_{\alpha, 7 \mathrm{\beta}}}=13, \mathrm{~J}_{7 \alpha, 8}=12.2$ & & \\
\hline & & 7-H $\beta$ & 1.94 & $\mathrm{~J}_{7 \beta, 6 \alpha}=2-4, \mathrm{~J}_{7 \beta, 8}=3.6$ & & \\
\hline C-8 & 40.6 & $8-\mathrm{H}$ & 2.14 & $\mathrm{~J}_{8,9}=12.2$ & 11,19 & \\
\hline C-9 & 42.3 & 9-H & 1.72 & $\mathrm{~J}_{9,11}=11.1$ & & \\
\hline $\mathrm{C}-10$ & 39.4 & & & & & \\
\hline $\mathrm{C}-11$ & 74.8 & $11-\mathrm{H}$ & 4.36 & & 18,19 & 12 \\
\hline $\mathrm{C}-12$ & 214.6 & & & & & \\
\hline $\mathrm{C}-13$ & 63.5 & & & & & \\
\hline C-14 & 85.8 & & & & & \\
\hline \multirow[t]{2}{*}{ C-15 } & 33.0 & $15-\mathrm{H}(\alpha$ or $\beta)$ & 1.78 & & & \\
\hline & & $15-\mathrm{H}(\alpha$ or $\beta)$ & 1.36 & & & \\
\hline \multirow[t]{2}{*}{$\mathrm{C}-16$} & 28.9 & $16-\mathrm{H}(\alpha$ or $\beta)$ & 1.73 & & 17,22 & \\
\hline & & $16-\mathrm{H}(\alpha$ or $\beta)$ & 2.00 & & & \\
\hline $\mathrm{C}-17$ & 41.9 & $17-\mathrm{H}$ & 4.11 & $\begin{array}{l}\mathrm{J}_{17,16(\alpha \text { or } \beta)}=7.4, \\
J_{17,16(\alpha \text { or } \beta)}=9.7\end{array}$ & & $\begin{array}{c}12,13,20,21 \\
22\end{array}$ \\
\hline $\mathrm{C}-18$ & 18.0 & $18-\mathrm{CH}_{3}$ & 0.90 & & 22 & $12,13,14,17$ \\
\hline $\mathrm{C}-19$ & 23.7 & $19-\mathrm{CH}_{3}$ & 1.20 & & & $1,5,9,10$ \\
\hline $\mathrm{C}-20$ & 123.0 & & & & & \\
\hline $\mathrm{C}-21$ & 151.4 & 21-H & 7.51 & $\mathrm{~J}_{21,22}=2.6$ & & 20,24 \\
\hline $\mathrm{C}-22$ & 148.9 & $22-\mathrm{H}$ & 7.91 & $\mathrm{~J}_{22,23}=9.8$ & & 24 \\
\hline $\mathrm{C}-23$ & 115.7 & $23-\mathrm{H}$ & 6.31 & & & 20,24 \\
\hline $\mathrm{C}-24$ & 164.3 & & & & & \\
\hline $\mathrm{C}-1$ & 107.2 & $1 '-H$ & 4.35 & $\mathrm{~J}_{1^{\prime}, 2^{\prime}}=7.5$ & 5 'ax & 3 \\
\hline$C-2$ & 75.3 & $2 '-H$ & 3.25 & $\mathbf{J}_{2^{2}, 3^{\prime}}=9.1$ & & \\
\hline C-3' & 77.8 & $3 '-\mathrm{H}$ & 3.32 & $\mathrm{~J}_{3,4}, 4=8.7$ & & \\
\hline C-4' & 70.9 & 4'-H & 3.48 & $\mathrm{~J}_{4,5} 5^{\prime} \mathrm{ax}=10.4, \mathrm{~J}_{4,5^{\prime} \mathrm{eq}}=5.4$ & & \\
\hline \multirow[t]{2}{*}{$\mathrm{C}-5$} & 66.9 & $5^{\prime}-\mathrm{H}_{\mathrm{ax}}$ & 3.18 & $\mathrm{~J}_{5^{\prime} \mathrm{ax}, 5^{\prime} \mathrm{eq}}=11.4$ & & \\
\hline & & $5^{\prime}-\mathrm{H}_{\mathrm{eq}}$ & 3.83 & & & \\
\hline
\end{tabular}




\section{Compound 11}

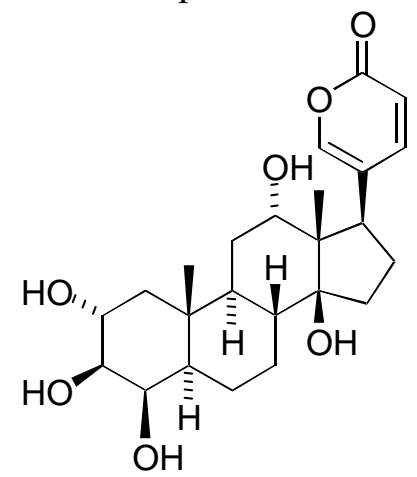

\begin{tabular}{|c|c|c|c|c|c|c|}
\hline $\begin{array}{l}\text { Carbon } \\
\text { No. }\end{array}$ & $\delta, \mathrm{ppm}$ & Proton No. & $\delta, \mathrm{ppm}$ & $J, \mathbf{H z}$ & $\begin{array}{c}\text { NOESY } \\
\text { Correlations }\end{array}$ & $\begin{array}{c}\text { HMBC } \\
\text { Correlations }\end{array}$ \\
\hline \multirow[t]{2}{*}{$\mathrm{C}-1$} & 46.8 & $1-\mathrm{H} \alpha$ & 1.02 & $\mathrm{~J}_{1 \alpha, 1 \beta}=12, \mathrm{~J}_{1 \alpha, 2}=12$ & 9 & \\
\hline & & $1-\mathrm{H} \beta$ & 1.96 & $\mathrm{~J}_{1 \beta, 2}=4.4$ & & $2,3,5,10,19$ \\
\hline $\mathrm{C}-2$ & 69.2 & $2-\mathrm{H}$ & 3.83 & $\mathrm{~J}_{2,3}=10$ & 19 & 3 \\
\hline $\mathrm{C}-3$ & 78.5 & $3-\mathrm{H}$ & 3.27 & $\mathrm{~J}_{3,4}=2.7$ & & 2 \\
\hline C-4 & 76.3 & 4-H & 3.81 & $\mathrm{~J}_{4,5}=3.3$ & & 2,10 \\
\hline$C-5$ & 50.0 & $5-\mathrm{H}$ & 1.16 & $\mathrm{~J}_{5,6 \alpha}=3, \mathrm{~J}_{5,6 \mathrm{~B}}=12.5$ & 9 & \\
\hline \multirow[t]{2}{*}{ C-6 } & 26.5 & 6-Ha & 1.47 & $\mathrm{~J}_{6 \alpha, 6 \beta}=12.5, \mathrm{~J}_{6 \alpha, 7 \alpha}=3$ & & \\
\hline & & $6-\mathrm{H} \beta$ & 1.75 & $\mathrm{~J}_{6 \beta, 7 \alpha}=12.5, \mathrm{~J}_{6 \beta, 7 \beta}=3.5$ & 19 & \\
\hline \multirow[t]{2}{*}{$\mathrm{C}-7$} & 28.8 & $7-\mathrm{H \alpha}$ & 1.22 & $\mathrm{~J}_{7 \alpha, 7 \beta}=12.5, \mathrm{~J}_{7 \alpha, 8}=12$ & & \\
\hline & & 7-H $\beta$ & 2.15 & $\mathrm{~J}_{7 \beta, 8}=3.7$ & & \\
\hline C-8 & 42.3 & $8-\mathrm{H}$ & 1.60 & $\mathrm{~J}_{8,9}=12$ & $11 \beta, 18$ & \\
\hline C-9 & 45.2 & $9-\mathrm{H}$ & 1.38 & $\mathrm{~J}_{9,11 \alpha}=3.2, \mathrm{~J}_{9,11 \beta}=12$ & $15 \alpha$ & \\
\hline $\mathrm{C}-10$ & 38.2 & & & & & \\
\hline \multirow[t]{2}{*}{$\mathrm{C}-11$} & 29.5 & $11-\mathrm{H} \alpha$ & 1.61 & $\mathrm{~J}_{11 \alpha, 11 \beta}=12, \mathrm{~J}_{11 \alpha, 12}=3$ & & \\
\hline & & $11-\mathrm{H} \beta$ & 1.53 & $\mathrm{~J}_{11 \beta, 12}=3$ & 18,19 & \\
\hline $\mathrm{C}-12$ & 76.4 & $12-\mathrm{H}$ & 3.57 & & 17,18 & 9,14 \\
\hline $\mathrm{C}-13$ & 53.3 & & & & & \\
\hline $\mathrm{C}-14$ & 86.1 & & & & & \\
\hline \multirow[t]{2}{*}{$\mathrm{C}-15$} & 34.6 & $15-\mathrm{H}(\alpha$ or $\beta)$ & 2.16 & & & \\
\hline & & $15-\mathrm{H}(\alpha$ or $\beta)$ & 1.60 & & & \\
\hline \multirow[t]{2}{*}{$\mathrm{C}-16$} & 31.4 & $16-\mathrm{H}(\alpha$ or $\beta)$ & 1.64 & & & \\
\hline & & $16-\mathrm{H}(\alpha$ or $\beta)$ & 2.07 & & & 14 \\
\hline $\mathrm{C}-17$ & 46.0 & $17-\mathrm{H}$ & 3.20 & $\begin{array}{l}\mathrm{J}_{17,16(\alpha \text { or } \beta)}=6, \\
\mathrm{~J}_{17,16(\alpha \text { or } \beta)}=10\end{array}$ & 21 & $\begin{array}{c}12,13,14,16 \\
20\end{array}$ \\
\hline C-18 & 18.5 & $18-\mathrm{CH}_{3}$ & 0.66 & & & $12,13,14,17$ \\
\hline C-19 & 16.2 & 19- $\mathrm{CH}_{3}$ & 1.08 & & & $1,5,9,10$ \\
\hline $\mathrm{C}-20$ & 125.8 & & & & & \\
\hline $\mathrm{C}-21$ & 150.3 & 21-H & 7.40 & $\mathrm{~J}_{21,22}=2.6$ & & $20,22,24$ \\
\hline $\mathrm{C}-22$ & 149.7 & $22-\mathrm{H}$ & 8.01 & $\mathrm{~J}_{22,23}=9.6$ & & 21,24 \\
\hline $\mathrm{C}-23$ & 115.4 & $23-\mathrm{H}$ & 6.28 & & & 20,24 \\
\hline C-24 & 164.2 & & & & & \\
\hline
\end{tabular}




\section{Compound 12}

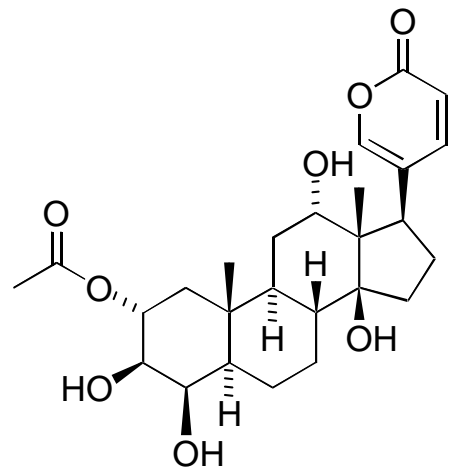

\begin{tabular}{|c|c|c|c|c|c|c|}
\hline $\begin{array}{l}\text { Carbon } \\
\text { No. }\end{array}$ & $\delta, \mathrm{ppm}$ & Proton No. & $\delta, \mathrm{ppm}$ & $J, \mathrm{~Hz}$ & $\begin{array}{c}\text { NOESY } \\
\text { Correlations }\end{array}$ & $\begin{array}{c}\text { HMBC } \\
\text { Correlations }\end{array}$ \\
\hline \multirow[t]{2}{*}{$\mathrm{C}-1$} & 43.6 & $1-\mathrm{H} \alpha$ & 1.08 & $\mathrm{~J}_{1 \alpha, 1 \beta}=12, \mathrm{~J}_{1 \alpha, 2}=11$ & 3 & \\
\hline & & $1-\mathrm{H} \beta$ & 2.00 & $\mathrm{~J}_{1 \beta, 2}=4.6$ & & 10,19 \\
\hline $\mathrm{C}-2$ & 73.5 & $2-\mathrm{H}$ & 5.13 & $\mathrm{~J}_{2,3}=11$ & 19 & $1^{\prime}, 3$ \\
\hline C-3 & 75.3 & $3-\mathrm{H}$ & 3.52 & $\mathrm{~J}_{3,4}=3.5$ & 5 & 2 \\
\hline C-4 & 76.1 & $4-\mathrm{H}$ & 3.86 & $\mathrm{~J}_{4,5}=4.5$ & & 2 \\
\hline C-5 & 49.6 & $5-\mathrm{H}$ & 1.20 & $\mathrm{~J}_{5,6 \mathrm{\alpha}}=3, \mathrm{~J}_{5,6 \mathrm{~B}}=12.5$ & & \\
\hline \multirow[t]{2}{*}{ C-6 } & 26.3 & 6-H $\alpha$ & 1.48 & $\mathrm{~J}_{6 \alpha, 6 \beta}=12.5, \mathrm{~J}_{6 \alpha, 7 \alpha}=3$ & & \\
\hline & & $6-\mathrm{H} \beta$ & 1.77 & $\mathrm{~J}_{6 \beta, 7 \alpha}=12.5, \mathrm{~J}_{6 \beta, 7 \beta}=3$ & 19 & \\
\hline \multirow[t]{2}{*}{$\mathrm{C}-7$} & 28.7 & 7-Ha & 1.22 & $\mathrm{~J}_{7 \alpha, 7 \beta}=12.5, \mathrm{~J}_{7 \alpha, 8}=12.5$ & & \\
\hline & & 7-H $\beta$ & 2.16 & $\mathrm{~J}_{7 \beta, 8}=3.2$ & & \\
\hline C-8 & 42.1 & $8-\mathrm{H}$ & 1.60 & $\mathrm{~J}_{8,9}=12$ & 18 & \\
\hline C-9 & 45.0 & 9-H & 1.38 & & & \\
\hline $\mathrm{C}-10$ & 38.1 & & & & & \\
\hline \multirow[t]{2}{*}{$\mathrm{C}-11$} & 29.4 & $11-\mathrm{H} \alpha$ & 1.52 & & & \\
\hline & & $11-\mathrm{H} \beta$ & 1.53 & & 19,18 & \\
\hline $\mathrm{C}-12$ & 76.2 & $12-\mathrm{H}$ & 3.56 & $\mathrm{~J}_{11 \beta, 12}=3, \mathrm{~J}_{11 \alpha, 12}=3$ & 18 & 9,14 \\
\hline $\mathrm{C}-13$ & 53.1 & & & & & \\
\hline $\mathrm{C}-14$ & 85.8 & & & & & \\
\hline \multirow[t]{2}{*}{$\mathrm{C}-15$} & 34.5 & $15-\mathrm{H}(\alpha$ or $\beta)$ & 2.16 & & & \\
\hline & & $15-\mathrm{H}(\alpha$ or $\beta)$ & 1.59 & & & \\
\hline \multirow[t]{2}{*}{$\mathrm{C}-16$} & 31.3 & $16-\mathrm{H}(\alpha$ or $\beta)$ & 1.64 & & & \\
\hline & & $16-\mathrm{H}(\alpha$ or $\beta)$ & 2.06 & & & \\
\hline $\mathrm{C}-17$ & 45.9 & $17-\mathrm{H}$ & 3.19 & $\begin{aligned} \mathrm{J}_{17,16(\alpha \text { or } \beta)}=6.5 \\
\mathrm{~J}_{17,16(\alpha \text { or } \beta)}=10\end{aligned}$ & 21 & $13,16,21$ \\
\hline $\mathrm{C}-18$ & 18.4 & $18-\mathrm{CH}_{3}$ & 0.66 & & & $12,13,14,17$ \\
\hline $\mathrm{C}-19$ & 15.5 & $19-\mathrm{CH}_{3}$ & 1.14 & & & $1,5,9,10$ \\
\hline $\mathrm{C}-20$ & 125.5 & & & & & \\
\hline $\mathrm{C}-21$ & 150.3 & 21-H & 7.40 & $\mathrm{~J}_{21,22}=2.4$ & & $17,20,22,24$ \\
\hline $\mathrm{C}-22$ & 149.7 & $22-\mathrm{H}$ & 8.01 & $\mathrm{~J}_{22,23}=9.7$ & & 21,24 \\
\hline $\mathrm{C}-23$ & 115.4 & $23-\mathrm{H}$ & 6.29 & & & 20,24 \\
\hline $\mathrm{C}-24$ & 164.7 & & & & & \\
\hline $\mathrm{C}-1^{\prime}$ & 172.9 & & & & & \\
\hline $\mathrm{C}-2^{\prime}$ & 21.1 & $2 '-\mathrm{CH}_{3}$ & 2.05 & & & 1 ' \\
\hline
\end{tabular}




\section{Compound 13}

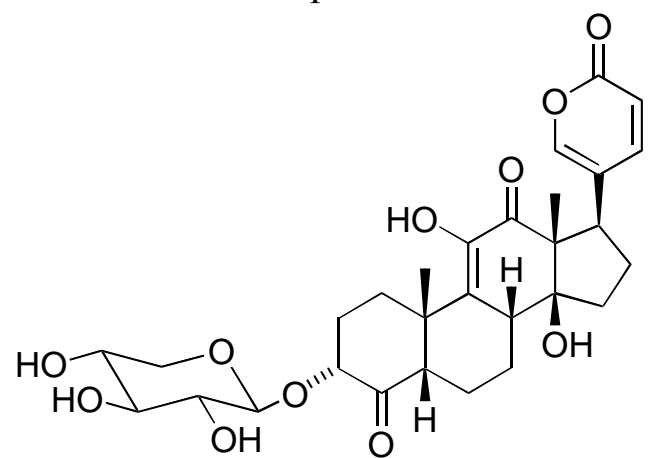

\begin{tabular}{|c|c|c|c|c|c|c|}
\hline $\begin{array}{c}\text { Carbon } \\
\text { No. }\end{array}$ & $\delta, \mathrm{ppm}$ & Proton No. & $\delta, \mathrm{ppm}$ & $J, \mathrm{~Hz}$ & $\begin{array}{c}\text { NOESY } \\
\text { Correlations }\end{array}$ & $\begin{array}{c}\text { HMBC } \\
\text { Correlations }\end{array}$ \\
\hline \multirow[t]{2}{*}{$\mathrm{C}-1$} & 30.5 & $1-\mathrm{H} \alpha$ & 2.44 & $\mathrm{~J}_{1 \alpha, 1 \beta}=9.5, \mathrm{~J}_{1 \alpha, 2 \alpha}=3-4$ & & \\
\hline & & $1-\mathrm{H} \beta$ & 2.26 & $\mathrm{~J}_{1 \beta, 2 \alpha}=12$ & & \\
\hline \multirow[t]{2}{*}{$\mathrm{C}-2$} & 30.4 & $2-\mathrm{H}$ & 2.23 & $J_{2 \alpha, 2 \beta}=12, J_{2 \alpha, 3}=9.0$ & & \\
\hline & & $2-\mathrm{H}$ & 1.88 & $\mathrm{~J}_{2 \beta, 1 \alpha}=3-4, \mathrm{~J}_{2 \beta, 3}=6.1$ & & \\
\hline $\mathrm{C}-3$ & 79.8 & $3-\mathrm{H}$ & 4.50 & & 1 ' & 1 ' \\
\hline $\mathrm{C}-4$ & 213.5 & & & & & \\
\hline C-5 & 55.6 & $5-\mathrm{H}$ & 2.65 & $\mathrm{~J}_{5,6 \alpha}=7.2, \mathrm{~J}_{5,6 \beta}=7.2$ & 19 & 4,9 \\
\hline \multirow[t]{2}{*}{ C-6 } & 22.7 & 6-H $\alpha$ & 2.09 & $\mathrm{~J}_{6 \alpha, 6 \beta}=12, \mathrm{~J}_{6 \alpha, 7 \alpha}=2-4$ & & \\
\hline & & $6-\mathrm{H} \beta$ & 1.93 & $\mathrm{~J}_{6 \beta, 7 \alpha}=11, \mathrm{~J}_{6 \beta, 7 \beta}=2-4$ & & \\
\hline \multirow[t]{2}{*}{$\mathrm{C}-7$} & 22.5 & 7-Ha & 1.60 & $\mathrm{~J}_{7 \alpha, 7 \beta}=12.5, \mathrm{~J}_{7 \alpha, 8}=12.5$ & & \\
\hline & & $7-\mathrm{H} \beta$ & 2.19 & $\mathrm{~J}_{7 \beta, 8}=4.5$ & & \\
\hline $\mathrm{C}-8$ & 40.4 & $8-\mathrm{H}$ & 2.77 & & 19,18 & 9,11 \\
\hline C-9 & 133.6 & & & & & \\
\hline $\mathrm{C}-10$ & 43.8 & & & & & \\
\hline $\mathrm{C}-11$ & 141.9 & & & & & \\
\hline $\mathrm{C}-12$ & 201.3 & & & & & \\
\hline $\mathrm{C}-13$ & 60.3 & & & & & \\
\hline C-14 & 82.9 & & & & & \\
\hline \multirow[t]{2}{*}{$C-15$} & 33.0 & $15-\mathrm{H}(\alpha$ or $\beta)$ & 1.76 & & & \\
\hline & & $15-\mathrm{H}(\alpha$ or $\beta)$ & 1.30 & & & \\
\hline \multirow[t]{2}{*}{ C-16 } & 28.8 & $16-\mathrm{H}(\alpha$ or $\beta)$ & 1.76 & & & \\
\hline & & $16-\mathrm{H}(\alpha$ or $\beta)$ & 1.99 & & & \\
\hline $\mathrm{C}-17$ & 44.7 & $17-\mathrm{H}$ & 3.81 & $\begin{array}{l}\mathrm{J}_{17,16(\alpha \text { o o } \beta)}=7, \\
\mathrm{~J}_{17,16(\alpha \text { or } \beta)}=9\end{array}$ & & \\
\hline $\mathrm{C}-18$ & 16.9 & $18-\mathrm{CH}_{3}$ & 0.81 & & 8 & $12,13,14,17$ \\
\hline C-19 & 25.6 & $19-\mathrm{CH}_{3}$ & 1.34 & & 5,8 & $1,5,9,10$ \\
\hline C-20 & 122.9 & & & & & \\
\hline C-21 & 151.7 & 21-H & 7.59 & & & 17 \\
\hline C- 22 & 148.9 & $22-\mathrm{H}$ & 7.96 & & & 17 \\
\hline C-23 & 115.7 & $23-\mathrm{H}$ & 6.32 & & & \\
\hline C-24 & 164.3 & & & & & \\
\hline C-1' & 104.3 & $1^{\prime}-\mathrm{H}$ & 4.25 & & 3 & \\
\hline C-2' & 74.9 & $2^{\prime}-\mathrm{H}$ & 3.23 & & & \\
\hline C-3' & 77.6 & $3^{\prime}-\mathrm{H}$ & 3.31 & & & \\
\hline C-4' & 70.9 & 4'-H & 3.48 & & & \\
\hline \multirow[t]{2}{*}{$\mathrm{C}-5$} & 66.9 & $5^{\prime}-\mathrm{H}_{\mathrm{ax}}$ & 3.16 & & & \\
\hline & & $5^{\prime}-\mathrm{H}_{\mathrm{eq}}$ & 3.83 & & & \\
\hline
\end{tabular}

